\title{
Mesiodistal Size Asymmetry of the Left and Right Quadrant
}

\author{
Navid Naseri, ${ }^{1}$ Pedram Baghaeian, ${ }^{2}$ Maryam Javaherimahd, ${ }^{2}$ and Fatemeh Gorjizadeh ${ }^{2,}$ \\ ${ }^{1}$ Orthodontic Department, School of Dentistry, Islamic Azad University, Shiraz, IR Iran \\ ${ }^{2}$ Orthodontic Department, Tehran University of Medical Sciences, Tehran, IR Iran \\ "Corresponding author: Fatemeh Gorjizadeh, Orthodontic Department, Tehran University of Medical Sciences, Tehran, IR Iran. E-mail: fatemegorjizade@gmail.com
}

Received 2015 December 30; Accepted 2016 January 30.

\begin{abstract}
Background: It has been proved that, there's craniofacial asymmetry but much of this skeletal asymmetries are clinically ignorable. This asymmetry may exist in teeth size arranged in right and left sides of human mouth too. Orthodontists should pay attention to bilateral tooth asymmetry in treatment planning stage.

Objectives: This study was conducted to demonstrate whether the difference between size of left and right side teeth is actual.

Materials and Methods: A total of 200 plaster dental molds were used which were collected from a private practice in Tehran. A caliper with accuracy of $0.01 \mathrm{~mm}$ was used for measuring teeth and most teeth were measured twice and the average value was considered as the teeth size. In all cases, the Vernier calipers jaws were moved along the teeth longitudinal axis and the biggest width was measured in the contact point area.

Results: In average $83.16 \%$ of left and right teeth in upper jaw and $83.66 \%$ of left and right teeth in the lower jaw were not symmetrical and teeth in the upper and lower jaws were completely similar $16.84 \%$ and $16.34 \%$, respectively.

Conclusions: The result of the study showed that nearly $83 \%$ of teeth in maxilla and mandible are asymmetric in mesiodistal width. Mandibular second molar and canine showed the highest and lowest bilateral asymmetry respectively.
\end{abstract}

Keywords: Asymmetry, Tooth Size, Mesiodistal Width

\section{Background}

It has been proved that, there's craniofacial asymmetry (1-4). There is a trivial asymmetry in right and left sides of human face, many studies have stated that the right side is bigger than the left side $(1,2,4,5)$. Much of this skeletal asymmetries are clinically ignorable, therefore it seems soft tissue tries to minimize underlying asymmetry (1). Considering this facial asymmetries, it seems that teeth arranged in right and left sides of human mouth might be asymmetric too.

Difference in one or more teeth size in the right and left sides in $90 \%$ of cases have been reported (6). Also it has been stated that the asymmetry of tooth size in right and left side would be due to congenital or environmental factors or both of them; All the asymmetries are divided in two classes: quantitative asymmetry or difference in number of teeth in each half-arch and qualitative asymmetry, which is due to difference in size of teeth mesiodistal width or their location in the dental arch (7).

\section{Objectives}

This study was conducted to demonstrate whether the difference between size of left and right side teeth is actual in the studied population.

\section{Materials and Methods}

In general, in this study 200 plaster casts (including 5600 teeth) of people who needed orthodontic treatment and had referred to an orthodontist were examined. A caliper with accuracy of $0.01 \mathrm{~mm}$ was used for measuring teeth. In this study the biggest mesiodistal width of all permanent teeth (except wisdom tooth) were measured (Figure 1). The samples were collected from a private practice in Tehran. Out of 200 samples prepared in this study, some samples were excluded because the gender of teeth owner was unknown; hence only 162 casts, male $(\mathrm{n}=39 ; 24.07 \%)$ and female $(\mathrm{n}=123 ; 75.92 \%)$ were considered. In this study, the following casts were considered:

1) Air-Bubble-free casts

2) Casts without any kind of transformation and extra plaster which may change the dental contour

3) The permanent teeth (except the wisdom teeth) must be erupted

4) Casts without severe crowding (severe crowding that might disrupt the measurements were excluded)

5) Without observable anomaly in tooth size: e.g. peg shape, macrodontia, microdontia, mesiodens

6) Without decay that has disturbed the dental contour, especially proximal decay 
7) Should not be with crown and bridge

8) Stone casts and casts without breakages and abrasion

9) Casts free from tooth rotations and inclinations that disturb measurements

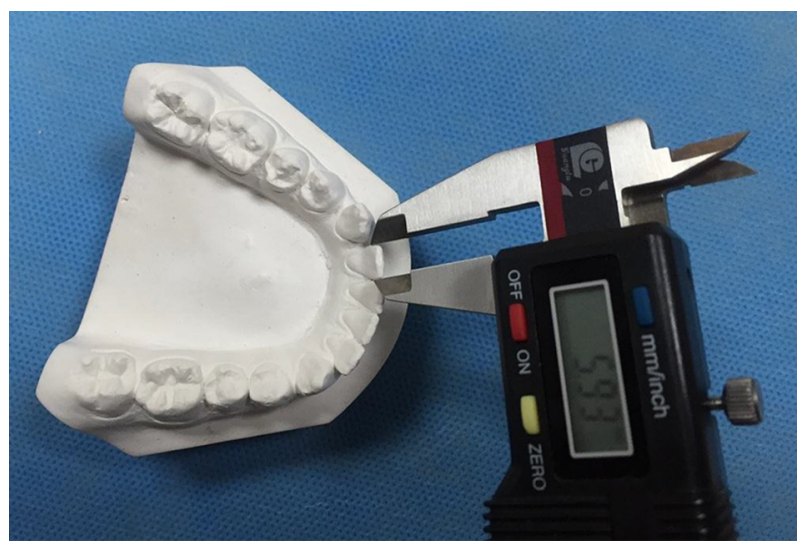

Figure 1. Vernier Digital Calipers was Used to Measure the Biggest Width of Tooth at the Contact Point Area

\subsection{Measurement Method}

A caliper with accuracy of $0.01 \mathrm{~mm}$ was used for measuring teeth and most teeth were measured twice and the average value was considered as the tooth size. In all cases, the vernier calipers' jaws were moved along with the teeth longitudinal axis and the biggest width was measured in the contact point area. For measuring the error, after the measurement was completed some teeth were chosen randomly through different parts of the mouth and were remeasured. The measurement difference varied from 0 to $0.04 \mathrm{~mm}$. The average value of difference was $0.016 \mathrm{~mm}$ with SD of $0.02 \mathrm{~mm}$, which statistically this amount of error with such SD is acceptable. Sample number, average size and SD were calculated for each tooth.

\section{Results}

Generally, in average $83.16 \%$ and $83.66 \%$ of right and left teeth arranged respectively in maxilla and mandible were not symmetrical; whereas only $16.84 \%$ and $16.34 \%$ of right and left side teeth respectively in maxilla and mandible were completely symmetrical (Tables 1 and 2 ).

Second molar and first premolar in maxilla and second molar and second premolar in mandible showed the highest difference in mesiodistal width in left and right sides (Tables 1 and 2).

Tables 3 and 4 show the average mesiodistal widths of teeth in maxilla and mandible.

\section{Discussion}

Analyzing old skulls, Woo indicated that there is a trivial asymmetry in right and left sides of human face, right side is bigger than left side which is due to more growth of brain in the right hemisphere (2). Shah and Jashi also confirmed the facial asymmetry due to bigger right side (1). In a study on anteroposterior cephalogram of 63 normal people showed that there is an asymmetry in all cases in which the left side was bigger, unlike the other studies (8). But much of this skeletal asymmetries are clinically ignorable, therefore it seems soft tissues try to minimize underlying asymmetry (1).

Regarding the trivial asymmetry in body's pair organs and also the trivial asymmetry in right and left sides of face, asymmetry is seen in dental sizes in both sides of the jaw arch, as a part of head and face hard tissue. As this study showed, nearly $83 \%$ of teeth in maxilla and mandible are asymmetric in mesiodistal width and there is no tendency for one side to be symmetrically larger in size. Many studies have shown the left and right tooth asymmetry and like us did not found any evidence for one side to be larger in size $(9,10)$.

This study also indicated that second molar and first premolar in maxilla and second molar and second premolar in mandible showed the highest difference in mesiodistal width in left and right sides (difference more than 0.25 $\mathrm{mm}$ ); of these, except upper first premolar the other are in accordance with findings of Garn et al. that the asymmetry is greater for the more distal tooth of each morphological class (9). We found the smallest asymmetry for lower canines while Garn et al. found the upper first premolar and also we found the greatest asymmetry for lower second molar as they did too (10). Ballard showed that the greatest asymmetry of mesiodistal width are present at lateral and first molar teeth in maxilla and canine and first premolar in the mandible (6).

Garn et al. showed that the asymmetry of maxillary teeth is a little greater than mandibular teeth (9), in this study, a little difference was seen in the asymmetry of mandibular and maxillary teeth.

In the cases of disharmony in tooth size, interproximal enamel reduction is a good technique for correction of discrepancies $(6,11-13)$.

\subsection{Conclusions}

The result of the study showed that nearly $83 \%$ of teeth in maxilla and mandible are asymmetric in mesiodistal width. Mandibular second molar and canine showed the highest and lowest bilateral asymmetry respectively. The 
Table 1. The Symmetry of Size of Right and Left Side Teeth in Maxilla

\begin{tabular}{lcccc}
\hline Tooth & No. & Without Difference, $\%$ & Difference up to o.25 mm, \% & Difference More Than o.25 mm, \% \\
\hline Middle incisors & 200 & 23.5 & 65 & 11.4 \\
\hline Lateral incisors & 197 & 12.22 & 74.6 & 13.2 \\
\hline Canine & 196 & 18.9 & 70.4 & 10.7 \\
\hline First premolar & 191 & 18.8 & 60.7 & 20.4 \\
\hline Second premolar & 194 & 17 & 70.3 & 16 \\
\hline First molar & 195 & 14.9 & 64 & 23.5 \\
\hline Second molar & 136 & 12.5 & & \\
\hline
\end{tabular}

Table 2. The Symmetry of Size of Right and Left Side Teeth in Mandible

\begin{tabular}{lcccc}
\hline Tooth & No & Without Difference, $\%$ & Difference up to o.25 mm, \% & Difference More Than 0.25 mm, \% \\
\hline Middle incisors & 200 & 28 & 62.5 & 9.5 \\
\hline Lateral incisors & 199 & 22.1 & 66.8 & 11.1 \\
\hline Canine & 200 & 8.9 & 82.8 & 7.3 \\
\hline First premolar & 190 & 17.9 & 69.5 & 11.1 \\
\hline Second premolar & 177 & 11.3 & 64.6 & 19.2 \\
\hline First molar & 189 & 19.6 & 65.6 & 27.9 \\
\hline Second molar & 122 & 6.6 & & \\
\hline
\end{tabular}

Table 3. Mean Value and SD of all Left and Right Teeth of Maxilla, Without Considering Gender

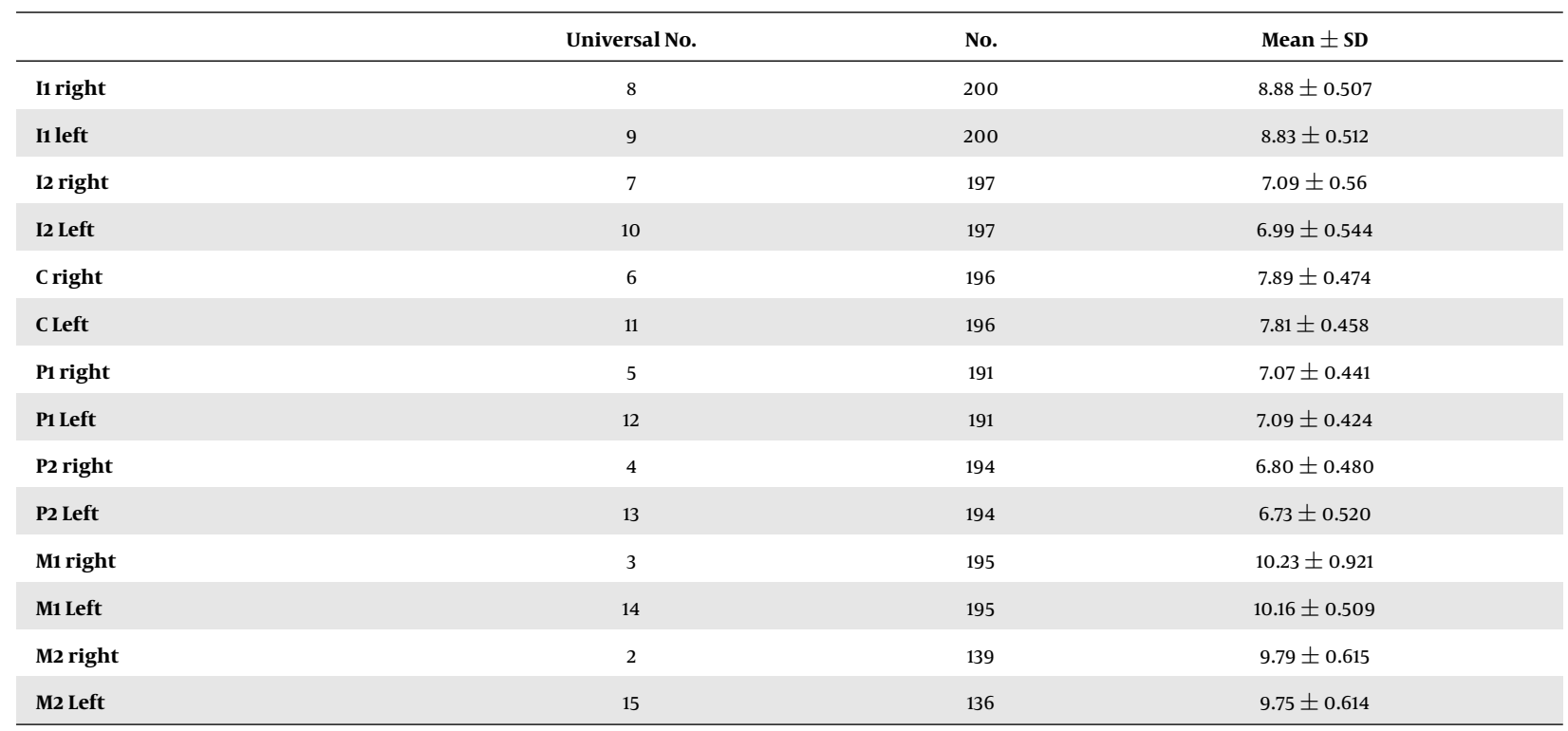

teeth sizes and the possible asymmetry must be determined at the beginning of orthodontic treatment. Stripping of proximal surfaces is a good solution for correcting the tooth size discrepancies.

\section{Acknowledgments}

Special thanks to Allahyar Geramy, professor of orthodontic department at Tehran University of medical science for his great scientific supports during this research. 
Table 4. Mean Value and SD of all Left and Right Teeth of Mandible, Without Considering Gender

\begin{tabular}{|c|c|c|c|}
\hline & Universal No. & No. & Mean \pm SD \\
\hline I1 right & 25 & 200 & $5.56 \pm 0.361$ \\
\hline I1 left & 24 & 200 & $5.57 \pm 0.355$ \\
\hline I2 right & 26 & 199 & $6.14 \pm 0.391$ \\
\hline I2 Left & 23 & 199 & $6.15 \pm 0.377$ \\
\hline C right & 27 & 200 & $6.90 \pm 0.432$ \\
\hline C Left & 22 & 200 & $6.87 \pm 0.458$ \\
\hline P1 right & 28 & 190 & $7.21 \pm 0.421$ \\
\hline P1 Left & 21 & 190 & $7.25 \pm 0.527$ \\
\hline P2 right & 29 & 177 & $7.17 \pm 0.574$ \\
\hline P2 Left & 20 & 177 & $7.21 \pm 0.478$ \\
\hline M1 right & 30 & 189 & $11.01 \pm 0.985$ \\
\hline M1 Left & 19 & 189 & $11.11 \pm 0.665$ \\
\hline M2 right & 31 & 122 & $10.25 \pm 0.609$ \\
\hline M2 Left & 18 & 122 & $10.24 \pm 0.735$ \\
\hline
\end{tabular}

\section{References}

1. Shah SM, Joshi MR. An assessment of asymmetry in the normal craniofacial complex. Angle Orthod. 1978;48(2):141-8. doi: 10.1043/00033219(1978)048<0141:AAOAIT>2.0.CO;2. [PubMed: 277077].

2. Woo TL. On the Asymmetry of the Human Skull. Biometrika. 1931;22(34):324-52. doi:10.1093/biomet/22.3-4.324.

3. Letzer GM, Kronman JH. A posteroanterior cephalometric evaluation of craniofacial asymmetry. Angle Orthod. 1967;37(3):205-11. doi: 10.1043/0003-3219(1967)037lt;0205:APCEOCgt;2.0.CO;2. [PubMed: 19919216].

4. Peck S, Peck L, Kataja M. Skeletal asymmetry in esthetically pleasing faces. Angle Orthod. 1991;61(1):43-8. doi: 10.1043/00033219(1991)061<0043:SAIEPF>2.0.CO;2. [PubMed: 2012321].

5. Ferrario VF, Sforza C, Poggio CE, Tartaglia G. Distance from symmetry: a three-dimensional evaluation of facial asymmetry. J Oral Maxillofac Surg. 1994;52(11):1126-32. [PubMed: 7965306].

6. Ballard ML. Asymmetry in tooth size: a factor in the etiology, diagnosis and treatment of malocclusion. Angle Orthod. 1944;14(3):67-70.
7. Lundström A. Some asymmetries of the dental arches, jaws, and skull, and their etiological significance. Am J Orthod. 1961;47(2):81-106. doi: 10.1016/0002-9416(61)90205-6.

8. Vig PS, Hewitt AB. Asymmetry of the human facial skeleton. Angle Orthod. 1975;45(2):125-9. doi: 10.1043/00033219(1975)045<0125:AOTHFS>2.0.CO;2. [PubMed: 1054939].

9. Garn SM, Lewis AB, Kerewsky RS. The meaning of bilateral asymmetry in the permanent dentition. Angle Orthod. 1966;36(1):55-62. doi: 10.1043/0003-3219(1966)036<0055:TMOBAI>2.0.CO;2. [PubMed: 5218762].

10. Garn SM, Lewis AB, Kerewsky RS. Buccolingual size asymmetry and its developmental meaning. Angle Orthod. 1967;37(3):186-93. doi: 10.1043/0003-3219(1967)037lt;0186:BSAAIDgt;2.0.CO;2. [PubMed: 19919214].

11. Tuverson DL. Anterior interocclusal relations. Part I. Am J Orthod. 1980;78(4):361-70. [PubMed: 6933846].

12. Livas C, Jongsma AC, Ren Y. Enamel reduction techniques in orthodontics: a literature review. Open Dent J. 2013;7:146-51. doi: 10.2174/1874210601307010146. [PubMed: 24265652].

13. Jarvis RG. Interproximal reduction in the molar/premolar region: the new approach. Aust Orthod J. 1990;11(4):236-40. [PubMed: 1967107]. 